\title{
Riemannian Geometry of Hartogs domains *
}

\author{
Antonio J. Di Scala, Andrea Loi, Fabio Zuddas
}

\begin{abstract}
Let $D_{F}=\left\{\left.\left(z_{0}, z\right) \in \mathbb{C}^{n}|| z_{0}\right|^{2}<b,\|z\|^{2}<F\left(\left|z_{0}\right|^{2}\right)\right\}$ be a strongly pseudoconvex Hartogs domain endowed with the Kähler metric $g_{F}$ associated to the Kähler form $\omega_{F}=-\frac{i}{2} \partial \bar{\partial} \log \left(F\left(\left|z_{0}\right|^{2}\right)-\|z\|^{2}\right)$.

This paper contains several results on the Riemannian geometry of these domains. These are summarized in Theorem 1.1, Theorem 1.2 and Theorem 1.3. In the first one we prove that if $D_{F}$ admits a non special geodesic (see definition below) through the origin whose trace is a straight line then $D_{F}$ is holomorphically isometric to an open subset of the complex hyperbolic space. In the second theorem we prove that all the geodesics through the origin of $D_{F}$ do not self-intersect, we find necessary and sufficient conditions on $F$ for $D_{F}$ to be geodesically complete and we prove that $D_{F}$ is locally irreducible as a Riemannian manifold. Finally, in Theorem 1.3, we compare the Bergman metric $g_{B}$ and the metric $g_{F}$ in a bounded Hartogs domain and we prove that if $g_{B}$ is a multiple of $g_{F}$, namely $g_{B}=\lambda g_{F}$, for some $\lambda \in \mathbb{R}^{+}$, then $D_{F}$ is holomorphically isometric to an open subset of the complex hyperbolic space.
\end{abstract}

Keywords: Kähler metrics; Hartogs domain; geodesics.

Subj.Class: 53C55, 32Q15, 32T15.

\section{Introduction}

Let $b \in \mathbb{R}^{+} \cup\{+\infty\}$ and let $F:[0, b) \rightarrow(0,+\infty)$ be a non increasing smooth function on $[0, b)$. The $n$-dimensional Hartogs domain $D_{F} \subset$ $\mathbb{C}^{n}$ associated to the function $F$ is defined by

$$
D_{F}=\left\{\left.\left(z_{0}, z\right) \in \mathbb{C}^{n}|| z_{0}\right|^{2}<b,\|z\|^{2}<F\left(\left|z_{0}\right|^{2}\right)\right\},
$$

where $z=\left(z_{1}, \ldots, z_{n-1}\right)$ and $\|z\|^{2}=\left|z_{1}\right|^{2}+\cdots+\left|z_{n-1}\right|^{2}$.

${ }^{*}$ This work was supported by the MIUR Project "Riemannian metrics and differentiable manifolds" 
Under the assumption that $D_{F}$ is strongly pseudoconvex one can prove (see Proposition 2.1 in [11]) that the natural $(1,1)$-form on $D_{F}$ given by

$$
\omega_{F}=-\frac{i}{2} \partial \bar{\partial} \log \left(F\left(\left|z_{0}\right|^{2}\right)-\|z\|^{2}\right)
$$

is a Kähler form on $D_{F}$ and this is equivalent to the requirement that $F$ satisfies the condition

$$
\left(\frac{x F^{\prime}}{F}\right)^{\prime}<0, \quad x \in[0, b),
$$

where the derivatives are taken with respect to the variable $x=\left|z_{0}\right|^{2}$. Notice that (3) (and hence the strongly pseudoconvexity of $D_{F}$ ) turns out to be equivalent to the strongly pseudoconvexity of the boundary of $D_{F}$ at all $z=\left(z_{0}, z_{1}, \ldots, z_{n-1}\right)$ with $\left|z_{0}\right|^{2}<x_{0}$ (see Proposition 2.1 in [11] for a proof). Denote by $g_{F}$ the Kähler metric associated to the Kähler form $\omega_{F}$. Throughout all this paper we assume that $D_{F}$ is equipped with this Kähler metric. Notice that when $F(x)=1-x, 0 \leq x<1, D_{F}$ equals the $n$-dimensional complex hyperbolic space $\mathbb{C} H^{n}$, namely the unit ball $B^{n}$ in $\mathbb{C}^{n}$ equipped with the hyperbolic metric $g_{h y p}=g_{F}$. Hartogs domains are interesting both from the mathematical and the physical point of view (see for example [4], 9] and [11] for the study of some Riemannian properties of $g_{F}$ and the Berezin quantization of $\left.\left(D_{F}, g_{F}\right), 1\right]$ and 10 for the construction of global symplectic coordinates on these domains and [12] for the construction of Kähler-Einstein metrics on Hartogs type domains on symmetric spaces).

In this paper we are interested in the Riemannian geometry of Hartogs domains. In particular we study the geodesics, the completeness and local irreducibility of such domains with respect to the metric $g_{F}$. We denote by $\mathcal{G}$ the set of those geodesics passing through the origin whose traces are straight lines of $\mathbb{C}^{n}$ intersected with $D_{F}$. Since the isometry group of $D_{F}$ contains $U(1) \times U(n-1)$, it is easily seen that the set $\mathcal{S}$ of geodesics of $D_{F}$ passing through the origin and contained in the plane $z_{0}=0$ or in the complex line $z_{1}=\cdots=z_{n-1}=0$ is included in $\mathcal{G}$. A geodesic $\ell \in \mathcal{G}$ will be called a special geodesic if it belongs to $\mathcal{S}$.

Our first result is the following interesting characterization of the complex hyperbolic space amongst Hartogs domains.

Theorem 1.1 Let $\left(D_{F}, g_{F}\right)$ be a Hartogs domain. If there exists $\ell \in \mathcal{G}$ such that $\ell \notin \mathcal{S}$, then $D_{F}$ is holomorphically isometric to an open subset of $\mathbb{C} H^{n}$.

In other words the previous theorem asserts that if there exists one non special geodesic $\ell$ through the origin of $D_{F}$ whose trace is a straight 
line, then $D_{F} \subset \mathbb{C} H^{n}$ (and hence, in this case, $\mathcal{G}$ coincide with the set of all the geodesic through the origin).

Our second result is the following:

Theorem 1.2 Let $\left(D_{F}, g_{F}\right)$ be a Hartogs domain. Then the following facts hold true.

(i) All the geodesics through the origin of $D_{F}$ do not self-intersect;

(ii) $D_{F}$ is geodesically complete with respect to the Kähler metric $g_{F}$ if and only if

$$
\left.\int_{0}^{\sqrt{b}} \sqrt{-\left(\frac{x F^{\prime}}{F}\right)^{\prime}}\right|_{x=u^{2}} d u=+\infty
$$

where we define $\sqrt{b}=+\infty$ for $b=+\infty$;

(iii) $\left(D_{F}, g_{F}\right)$ is locally irreducible around any of its points.

The first part of the previous theorem should be compared with the main result of D'Atri and Zhao [2] asserting that in a bounded homogeneous domain equipped with its Bergman metric all the geodesics do not intersect. (Notice that the homogeneous assumption for an Hartogs domain implies that $D_{F}$ is holomorphically equivalent to $B^{n}$, the unit ball in $\mathbb{C}^{n}$ (see e.g. Theorem 6.11 in [7).

Other properties of the geodesics of the Bergman metric can be found in [5] and [6]. In [5] Fefferman deeply studied the geodesics of the Bergman metric at the boundary points of a bounded domain $D$ while [6] is concerned with the existence of a closed geodesic in any non trivial homotopy class of a (non simply-connected) bounded domain. Regarding the completeness of the Bergman metric on a bounded domain, the reader is referred to the classical paper of S. Kobayashi 8 .

By the previous discussion it is natural to compare the Bergman metric $g_{B}$ and the metric $g_{F}$ on a bounded Hartogs domain. Our third and last result is the following:

Theorem 1.3 Let $D_{F}$ be a bounded Hartogs domain. Assume that $g_{B}$ is a multiple of $g_{F}$, namely $g_{B}=\lambda g_{F}$, for some $\lambda \in \mathbb{R}^{+}$. Then $g_{F}$ is Kähler-Einstein and therefore $D_{F}$ is holomorphically isometric to an open subset of $\mathbb{C} H^{n}$.

The first part of the proof of the previous theorem is an adaptation of the proof of the following (unpublished) proposition communicated by Miroslav Engliš to the second author and which deals with the more general class of generalized Hartogs domains.

Proposition 1.4 (Engliš) Let

$$
\widetilde{\Omega}=\left\{(z, w) \in \Omega \times \mathbb{C}^{k}:\|w\|^{2}<F(z)\right\}
$$


be a bounded and simply-connected generalized Hartogs domain, where $\Omega$ is a pseudoconvex domain in $\mathbb{C}^{n}$ and $-\log F$ is a smooth strictlyPSH function on $\Omega$. Let $g_{B}$ be the Bergman metric and let $g_{F}$ be the Kähler metric on $\widetilde{\Omega}$ whose Kähler potential is $-\log \left(F(z)-\|w\|^{2}\right)$. If $g_{B}=\lambda g_{F}$, for some $\lambda \in \mathbb{R}^{+}$, then $g_{F}$ is Kähler-Einstein.

The next section is dedicated to the proof of our theorems.

\section{Proof of the main results}

The following lemma is the main tool in the proofs of Theorem 1.1 and Theorem 1.2.

Lemma 2.1 Let $\left(D_{F}, g_{F}\right)$ be a Hartogs domain. Let $M \subset D_{F}$ be the real (plane) surface given by:

$$
M=D_{F} \cap\left\{\operatorname{Im}\left(z_{0}\right)=\operatorname{Im}\left(z_{1}\right)=0, z_{j}=0, j=2, \ldots, n-1\right\},
$$

and denote by $g$ the metric induced on $M$ by $g_{F}$. Then $(M, g)$ is totally geodesic, has constant Gaussian curvature equal to $-\frac{1}{2}$ and is geodesically complete if and only if condition (4) above is satisfied.

Proof: The surface $M$ is the fixed point set of the isometry of $D_{F}$ given by $\left(z_{0}, z_{1}, z_{2}, \ldots, z_{n-1}\right) \mapsto\left(\bar{z}_{0}, \bar{z}_{1},-z_{2}, \ldots,-z_{n-1}\right)$ and hence it is totally geodesic in $D_{F}$. By setting $u=\operatorname{Re}\left(z_{0}\right)$ and $v=\operatorname{Re}\left(z_{1}\right)$, this surface can be described as

$$
M=\left\{(u, v) \in \mathbb{R}^{2} \mid v^{2}<F\left(u^{2}\right), u^{2}<b\right\} .
$$

Furthermore, it is not difficult to see that the metric $g$ induced by $g_{F}$ on $M$ is given by

$$
g=\left(\begin{array}{ll}
g_{11} & g_{12} \\
g_{21} & g_{22}
\end{array}\right)=\frac{2}{\left(F-v^{2}\right)^{2}}\left(\begin{array}{cc}
C & -F^{\prime} u v \\
-F^{\prime} u v & F
\end{array}\right),
$$

where $C=F^{\prime 2} \cdot u^{2}-\left(F^{\prime}+F^{\prime \prime} \cdot u^{2}\right)\left(F-v^{2}\right)$ and $F$ and its derivatives are evaluated at $u^{2}$. By a straightforward, but long computation, one can verify that the Gaussian curvature of $g$ equals $-1 / 2$. Hence $(M, g)$ is isometric to an open subset, say $U$, of $\mathbb{R} H^{2}\left(-\frac{1}{2}\right)$, namely the unit disk $\left\{(x, y) \mid x^{2}+y^{2}<1\right\}$ in $\mathbb{R}^{2}$ endowed with the Beltrami-Klein metric

$$
g_{B K}=\frac{2}{\left(1-x^{2}-y^{2}\right)^{2}}\left[\left(1-y^{2}\right) d x^{2}+2 x y d x d y+\left(1-x^{2}\right) d y^{2}\right] .
$$

An isometry between $(M, g)$ and $U$ can be described explicitly. Indeed, let $\psi:(-\sqrt{b}, \sqrt{b}) \rightarrow \mathbb{R}$ be the strictly increasing real valued function defined by

$$
\psi(u)=\left.\int_{0}^{u} \sqrt{-\left(\frac{x F^{\prime}}{F}\right)^{\prime}}\right|_{x=s^{2}} d s .
$$


Then, it is not hard to see that the map

$$
\Psi: M \rightarrow \mathbb{R} H^{2}\left(-\frac{1}{2}\right),(u, v) \mapsto\left(\operatorname{Tanh}(\psi(u)), \frac{v}{\operatorname{Cosh}(\psi(u)) \sqrt{F\left(u^{2}\right)}}\right)
$$

is an injective local diffeomorphism satisfying $\Psi^{*}\left(g_{B K}\right)=g$. Therefore, the completeness of $M$ is equivalent to $\Psi(M)=\mathbb{R} H^{2}$, which is easily seen to be equivalent to condition (4), and we are done.

Remark 2.2 The fact that the surface $M$ in Lemma 2.1 is totally geodesic and that the isometry group of $D_{F}$ contains $U(1) \times U(n-1)$ implies the existence of an isometry of $D_{F}$, fixing the origin and taking any given geodesic passing through the origin of $D_{F}$ to a geodesic lying in $M$. This will be a key point in the proofs of both Theorem 1.1 and Theorem 1.2

Remark 2.3 All the $n$-dimensional Hartogs domain $D_{F}$ contains the complex totally geodesic surface

$$
\mathcal{B}=\left\{z_{j}=0, j=1, \ldots, n-1\right\} \cap D_{F},
$$

which in the literature of complex analysis is called the base of the Hartogs domain $D_{F}$. In view of the previous lemma, it is natural to consider the Hartogs domains where the Gaussian curvature of $\mathcal{B}$ is constant, , say equal to $K_{0}$,. It is not hard to see that such domains can be classified as follows:

(a) if $K_{0}=0$ then $F(t)=c e^{-k t}, c, k>0, t \in[0,+\infty)$, (complex analysts often refer to these domains as Spring domains);

(b) If $K_{0}>0$ then $F(t)=\left(c_{1}+c_{2} t\right)^{-\frac{2}{K_{0}}}$, with $c_{1}>0, c_{2}>0$, $t \in[0,+\infty)$

(c) If $K_{0}<0$ then $F(t)=\left(c_{1}+c_{2} t\right)^{-\frac{2}{K_{0}}}$, with $c_{1}>0, c_{2}<0$, $t \in\left[0,-\frac{c_{1}}{c_{2}}\right)$.

Notice that in the case (b), the corresponding Hartogs domain $D_{F}$ cannot be geodesically complete. In fact in this case also its base $\mathcal{B}$ would be complete and hence biholomorphic to $\mathbb{C} P^{1}$, yielding the contradiction $\mathcal{B} \cong \mathbb{C} P^{1} \subset D_{F} \subset \mathbb{C}^{n}$ (cfr. Example 2.6 at the end of the paper).

\section{Proof of Theorem 1.1.}

Let $\ell$ be a geodesic as in the statement of the theorem. Since $\ell \notin \mathcal{S}$, by Remark 2.2, we can assume $\ell \subset M$ and that

$$
\ell=\{v=k u, k \neq 0\} \cap M,
$$


where $u$ and $v$ are the parameters introduced in the proof of Lemma 2.1. Hence $\ell$ can be parametrized as $t \mapsto(u(t), v(t)=k u(t))$, where $t$ varies in a real interval containing the origin and the following geodesic equations have to be satisfied

$u^{\prime \prime}+\Gamma_{11}^{1} u^{\prime 2}+2 \Gamma_{12}^{1} u^{\prime} v^{\prime}+\Gamma_{22}^{1} v^{\prime 2}=0, v^{\prime \prime}+\Gamma_{11}^{2} u^{\prime 2}+2 \Gamma_{12}^{2} u^{\prime} v^{\prime}+\Gamma_{22}^{2} v^{\prime 2}=0$, namely

$$
\begin{gathered}
u^{\prime \prime}+\Gamma_{11}^{1} u^{\prime 2}+2 k \Gamma_{12}^{1} u^{\prime 2}+k^{2} \Gamma_{22}^{1} u^{\prime 2}=0 \\
k u^{\prime \prime}+\Gamma_{11}^{2} u^{\prime 2}+2 k \Gamma_{12}^{2} u^{\prime 2}+k^{2} \Gamma_{22}^{2} u^{\prime 2}=0,
\end{gathered}
$$

where $\Gamma_{j k}^{i}, i, j, k=1,2$ are the Christoffel symbols (see e.g. [3]). A straightforward computation gives :

$$
\begin{aligned}
& \Gamma_{11}^{1}=\frac{1}{2 D}\left(g_{22} \frac{\partial g_{11}}{\partial u}-g_{12}\left(2 \frac{\partial g_{12}}{\partial u}-\frac{\partial g_{11}}{\partial v}\right)\right)= \\
& =\frac{-4 u}{D\left(v^{2}-F\right)^{4}}\left[u^{2}\left(2 F^{\prime 2}+v^{2} F^{\prime \prime}\right)-F\left(v^{2}-F\right)\left(2 F^{\prime \prime}+u^{2} F^{\prime \prime \prime}\right)-F F^{\prime}\left(2 F^{\prime}+3 u^{2} F^{\prime \prime}\right)\right], \\
& \Gamma_{11}^{2}=\frac{1}{2 D}\left(-g_{12} \frac{\partial g_{11}}{\partial u}+g_{11}\left(2 \frac{\partial g_{12}}{\partial u}-\frac{\partial g_{11}}{\partial v}\right)\right)= \\
& =\frac{4 u^{2} v}{D\left(v^{2}-F\right)^{3}}\left[-u^{2} F^{\prime \prime 2}+F^{\prime}\left(F^{\prime \prime}+u^{2} F^{\prime \prime \prime}\right)\right] \text {, } \\
& \Gamma_{12}^{1}=\frac{1}{2 D}\left(g_{22} \frac{\partial g_{11}}{\partial v}-g_{12} \frac{\partial g_{22}}{\partial u}\right)= \\
& =\frac{-4 v}{D\left(v^{2}-F\right)^{4}}\left[-u^{2} F^{\prime 2}+F\left(F^{\prime}+u^{2} F^{\prime \prime}\right)\right], \\
& \Gamma_{12}^{2}=\frac{1}{2 D}\left(g_{11} \frac{\partial g_{22}}{\partial u}-g_{12} \frac{\partial g_{11}}{\partial v}\right)= \\
& =\frac{4 u F^{\prime}}{D\left(v^{2}-F\right)^{4}}\left[-u^{2} F^{\prime 2}+F\left(F^{\prime}+u^{2} F^{\prime \prime}\right)\right], \\
& \Gamma_{22}^{1}=\frac{1}{2 D}\left(-g_{12} \frac{\partial g_{22}}{\partial v}+g_{22}\left(2 \frac{\partial g_{12}}{\partial v}-\frac{\partial g_{22}}{\partial u}\right)\right)=0, \\
& \Gamma_{22}^{2}=\frac{1}{2 D}\left(g_{11} \frac{\partial g_{22}}{\partial v}-g_{12}\left(2 \frac{\partial g_{12}}{\partial v}-\frac{\partial g_{22}}{\partial u}\right)\right)= \\
& =\frac{-8 v}{D\left(v^{2}-F\right)^{4}}\left[-u^{2} F^{\prime 2}+F\left(F^{\prime}+u^{2} F^{\prime \prime}\right)\right],
\end{aligned}
$$


where

$$
D=g_{11} g_{22}-g_{12}^{2}=4 \frac{C F-F^{\prime 2} u^{2} v^{2}}{\left(F-v^{2}\right)^{4}} .
$$

By solving (10) with respect to $u^{\prime \prime}$ and substituting into (11) we get

$$
u^{\prime 2}\left[\Gamma_{11}^{2}+k\left(2 \Gamma_{12}^{2}-\Gamma_{11}^{1}\right)+k^{2}\left(\Gamma_{22}^{2}-2 \Gamma_{12}^{1}\right)-k^{3} \Gamma_{22}^{1}\right]=0
$$

Since $u^{\prime} \neq 0$ we get

$$
\Gamma_{11}^{2}+k\left(2 \Gamma_{12}^{2}-\Gamma_{11}^{1}\right)+k^{2}\left(\Gamma_{22}^{2}-2 \Gamma_{12}^{1}\right)-k^{3} \Gamma_{22}^{1}=0
$$

(where $\Gamma_{i j}^{k}=\Gamma_{i j}^{k}(u, k u)$ ).

By using (12) - (17), after a very long but straightforward calculation, the previous equation becomes

$$
\frac{8 k u\left(u^{4} F^{\prime \prime 2}+F\left(2 F^{\prime \prime}+u^{2} F^{\prime \prime \prime}\right)-F^{\prime}\left(2 u^{2} F^{\prime \prime}+u^{4} F^{\prime \prime \prime}\right)\right)}{D\left(k^{2} u^{2}-F\right)^{3}}=0,
$$

which, by setting $u^{2}=t, 0 \leq t<b$, is equivalent to the following ODE

$$
t^{2} F^{\prime \prime 2}+F\left(2 F^{\prime \prime}+t F^{\prime \prime \prime}\right)-F^{\prime}\left(2 t F^{\prime \prime}+t^{2} F^{\prime \prime \prime}\right)=0 .
$$

Notice that for $t \neq 0$ this equation can be written as

$$
t^{2} F^{\prime \prime 2}+\left(\frac{F}{t}-F^{\prime}\right)\left(t^{2} F^{\prime \prime}\right)^{\prime}=0 .
$$

By setting $G=t^{2} F^{\prime \prime}$ equation (22) becomes

$$
G^{\prime}=-\frac{F^{\prime \prime} t}{F-F^{\prime} t} G
$$

(notice that $F-F^{\prime} t>0$ for all $0<t<b$ since $F$ is not decreasing) and hence

$$
G(t)=c e^{\int \frac{-F^{\prime \prime} t}{F-F^{\prime} t} d t}=c\left(F-F^{\prime} t\right),
$$

for some $c \in \mathbb{R}$. For $t \rightarrow 0$ we get $c F(0)=0$, i.e. $c=0$. Therefore $G=t^{2} F^{\prime \prime}=0$, which implies $F(t)=c_{1}-c_{2} t$ for some $c_{1}, c_{2}>0$. Then the map

$$
\phi: D_{F} \rightarrow \mathbb{C} H^{n},\left(z_{0}, z_{1}, \ldots, z_{n-1}\right) \mapsto\left(\frac{z_{0}}{\sqrt{c_{1} / c_{2}}}, \frac{z_{1}}{\sqrt{c_{1}}}, \ldots, \frac{z_{n-1}}{\sqrt{c_{1}}}\right)
$$

is a holomorphic isometry of $D_{F}$ into an open subset of $\mathbb{C} H^{n}$ and this concludes the proof of Theorem 1.1. 
Remark 2.4 In the very definition of a Hartogs domain $D_{F}$ we have assumed (cfr. the introduction) that $F$ is non increasing in the interval $[0, b)$. The statement of Theorem 1.1 holds true also without this assumption. Indeed, it follows by condition (3) that $F^{\prime}(t)<0$ in a suitable interval $0 \leq t<\epsilon<b$, for some $\epsilon$, and the proof works also in this case.

\section{Proof of Theorem 1.2}

Let $\ell \subset D_{F}$ be a geodesic passing through the origin. By Remark 2.2 we can assume $\ell \subset M$. On the other hand by Lemma 2.1. $(M, g)$ is isometric to an open subset of $\mathbb{R} H^{2}\left(-\frac{1}{2}\right)$ where it is well-known that all the geodesics do not self intersect. This proves (i) of Theorem 1.2

Notice that, again by Remark 2.2 and by Hopf-Rinow's theorem the completeness of $g_{F}$ is equivalent to that of $g$, which by Lemma 2.1 is equivalent to (4) and we this proves (ii).

In order to prove (iii), assume by contradiction that $D_{F}$ is locally reducible around some point, say $p \in D_{F}$. Since the group $U(1) \times$ $U(n-1)$ acts by isometries on $\left(D_{F}, g_{F}\right)$ we can assume that $p \in M$ where $M$ is given by (5). So $p=(u, v, 0, \cdots, 0)$ and we can assume that both $u, v$ are (real numbers) different from zero (indeed if one of them is zero, say $p_{1}=0$, then $D_{F}$ is locally reducible around the point $p^{\prime}=\left(p_{1}^{\prime}, p_{2}, 0, \ldots 0\right)$ with $p_{1}^{\prime}$ sufficiently close to zero $)$. Therefore there exists an neighborhood $D \subset D_{F}$ of $p \in D_{F}$ such that $\left(D, g_{F}\right)$ splits as a Riemannian product i.e. $D=A \times B$, where $A$ and $B$ are Kähler manifolds. So the Lie algebra $\mathfrak{g}$ of Killing vector fields of $D$ also splits into two (or more) factors. Since $\mathfrak{u}(1) \times \mathfrak{u}(n-1) \subset \mathfrak{g}$ it follows that $\mathfrak{g}$ has at most two factors. Moreover since $p=(u, v, 0, \cdots, 0)$ with $u, v \neq 0$ we can recover the tangent space to the Riemannian factors $A$ and $B$. Thus, the factor $A$ is an open subset $A \subset \mathbb{C}$, with $u \in A$, and $B$ is an open subset $B \subset \mathbb{C}^{n-1}$, with $(v, 0, \cdots, 0) \in B$. In particular such Riemannian factors must be orthogonal w.r. to $g_{F}$. Then the coefficient $g_{12}$ of the metric $g$ on $M$ induced by $g_{F}$ has to be zero for $u$ and $v$ different from zero. On the other hand, by (7) above, $g_{12}=-F^{\prime} u v \neq 0$, a contradiction. This concludes the proof of (iii).

With the aid of (ii) in Theorem 1.2 we now study the completeness of two specific Hartogs domains.

Example 2.5 If $F(t)=c e^{-k t}, c, k>0, t \in[0,+\infty)$ then condition (4) is easily seen to be satisfied, so we get that the the Spring domains are complete (cfr. (a) of Remark 2.3).

Example 2.6 If $F(t)=\frac{1}{\left(c_{1}+c_{2} t\right)^{p}}\left(p \in \mathbb{N}^{+}\right), t \in[0,+\infty)$, then

$$
\left.\int_{0}^{\sqrt{b}} \sqrt{-\left(\frac{x F^{\prime}}{F}\right)^{\prime}}\right|_{x=u^{2}} d u=\int_{0}^{+\infty} \frac{\sqrt{c_{1} c_{2} p}}{c_{1}+c_{2} u^{2}} d u=\frac{\pi}{2} \sqrt{p}<\infty
$$


which proves that, for such $F$, the domain $D_{F}$ is not complete (cfr. (b) of Remark 2.3).

We now prove the last result of this paper.

\section{Proof of Theorem 1.3}

Recall that the Bergman metric $g_{B}$ on $D_{F}$ is, by definition, the one given by the Kähler potential $\log \widetilde{K}\left(z_{0}, z ; z_{0}, z\right)$, where $\widetilde{K}\left(z_{0}, z ; z_{0}^{\prime}, z^{\prime}\right)$ is the Bergman kernel of $D_{F}$. Let

$$
\tilde{F}\left(z_{0}, z\right):=F\left(\left|z_{0}\right|^{2}\right)-\|z\|^{2} .
$$

Note that this is a local defining function (positively signed) for $D_{F}$ at any boundary point $\left(z_{0}, z\right)$ with $z \in \mathcal{B}$, and such boundary points are strictly pseudoconvex. The hypothesis of the theorem and the fact that $D_{F}$ is contractible means that

$$
\log \widetilde{K}\left(z_{0}, z\right)=-\lambda \log \widetilde{F}\left(z_{0}, z\right)+2 \operatorname{Re} G\left(z_{0}, z\right)
$$

for some holomorphic function $G$ on $D_{F}$; here and below we will write just $\widetilde{K}\left(z_{0}, z\right)$ for $\widetilde{K}\left(z_{0}, z ; z_{0}, z\right)$. By rotational symmetry of $\widetilde{K}$ and $F$, the pluriharmonic function $2 \operatorname{Re} G$ must depend only on $\left|z_{0}\right|^{2}$ and $\|z\|^{2}$, hence must be a positive constant, say $\mu$. Thus

$$
\widetilde{K}\left(z_{0}, z\right)=\frac{\mu}{\widetilde{F}\left(z_{0}, z\right)^{\lambda}} .
$$

On the other hand, by Fefferman's formula [5] for the boundary singularity of the Bergman kernel,

$$
\widetilde{K}\left(z_{0}, z\right)=\frac{a\left(z_{0}, z\right)}{\widetilde{F}\left(z_{0}, z\right)^{n+1}}+b\left(z_{0}, z\right) \log \widetilde{F}\left(z_{0}, z\right), \quad\left(z_{0}, z\right) \in D_{F}
$$

where $a, b \in C^{\infty}\left(\mathcal{B} \times \mathbb{C}^{n-1}\right)$ and

$$
a\left(z_{0}, z\right)=\frac{n !}{\pi^{n}} J[\tilde{F}]\left(z_{0}, z\right),
$$

for $z_{0} \in \mathcal{B}$ and $\|z\|^{2}=F\left(\left|z_{0}\right|^{2}\right)$ and where $J[\widetilde{F}]$ is the Monge-Ampere determinant

$$
J[\widetilde{F}]=(-1)^{n} \operatorname{det}\left[\begin{array}{ccc}
\widetilde{F} & \frac{\partial \widetilde{F}}{\partial z_{0}} & \partial_{z} \widetilde{F} \\
\frac{\partial \widetilde{F}}{\partial \bar{z}_{0}} & \frac{\partial^{2} F}{\partial z_{0} \partial \bar{z}_{0}} & \partial_{z}\left(\frac{\partial \widetilde{F}}{\partial \bar{z}_{0}}\right) \\
\partial_{\bar{z}} \widetilde{F} & \partial_{\bar{z}}\left(\frac{\partial \widetilde{F}}{\partial \bar{z}_{0}}\right) & \partial_{\bar{z}} \partial_{z} \widetilde{F}
\end{array}\right] .
$$

A direct computation gives

$$
J[\widetilde{F}]=-F^{2} \frac{\partial^{2} \log F}{\partial z_{0} \partial \bar{z}_{0}} .
$$


(which depends only on $\left|z_{0}\right|^{2}$ ). By comparing (26) with (27) one gets:

$\mu=\frac{a\left(z_{0}, z\right) \widetilde{F}\left(z_{0}, z\right)^{\lambda}}{\widetilde{F}\left(z_{0}, z\right)^{n+1}}+b\left(z_{0}, z\right) \widetilde{F}\left(z_{0}, z\right)^{\lambda} \log \widetilde{F}\left(z_{0}, z\right), \quad\left(z_{0}, z\right) \in D_{F}$,

which evaluated at $\|z\|^{2}=F\left(\left|z_{0}\right|^{2}\right)$, forces $\lambda=n+1$. Further, by (28) and (29), the last expression gives

$$
-F^{2} \frac{\partial^{2} \log F}{\partial z_{0} \bar{\partial} z_{0}}=c,
$$

for all $z_{0} \in \mathcal{B}$ and $\|z\|^{2}=F\left(\left|z_{0}\right|^{2}\right)$, where $c$ is the negative constant given by $c=-\frac{\mu \pi^{n}}{n !}$ (notice that the condition $\|z\|^{2}=F\left(\left|z_{0}\right|^{2}\right.$ ) is superfluous, since nothing there depends on $z$ ). Feeding this back into formula (29) one gets $J[F]\left(z_{0}, z\right)=c$ for all $\left(z_{0}, z\right) \in D_{F}$, i.e. $g_{F}$ is Kähler-Einstein.

Let us recall now Lemma 3.1. of [11].

Lemma 2.7 Let $\left(D_{F}, g_{F}\right)$ be an n-dimensional Hartogs domain. Assume that one of its generalized scalar curvatures is constant. Then $\left(D_{F}, g_{F}\right)$ is holomorphically isometric to an open subset of the $n$ dimensional complex hyperbolic space.

Since the scalar curvature is one of the generalized scalar curvatures the proof of Theorem 1.3 is complete.

\section{References}

[1] F. Cuccu and A. Loi, Global symplectic coordinates on complex domains, J. Geom. and Phys. 56 (2006), 247-259.

[2] J. E. D'Atri and Y. D. Zhao, Geodesics and Jacobi fields in bounded homogeneous domains, Proc. Amer. Math. Soc. 89 no.. 1 (1983), 55-61.

[3] M. P. Do Carmo, Riemannian Geometry, Birkhäuser 1992.

[4] M. Engliš, Berezin Quantization and Reproducing Kernels on Complex Domains, Trans. Amer. Math. Soc. vol. 348 (1996), 411479.

[5] C. Fefferman, The Bergman kernel and biholomorphic mappings of pseudoconvex domains, Invent. Math. 26 (1974), 1-65.

[6] G. Herbort, On the geodesics of the Bergman metric, Math. Ann. 264 no. 1 (1983), 39-51.

[7] A. V. Isaev and S. G. Krantz, Domains with non-compact automorphism group: a survey, Adv. Math. 146 no. 1 (1999), 1-38. 
[8] S. Kobayashi, Geometry of bounded domains, Trans. Amer. Math. Soc. vol. 92 (1959), pp. 267-290.

[9] A. Loi, Regular quantizations of Kähler manifolds and constant scalar curvature metrics, J. Geom. and Phys. 53 (2005), 354-364.

[10] A. Loi and F. Zuddas, Symplectic maps of complex domains into complex space forms, preprint (2007).

[11] A. Loi and F. Zuddas, Extremal metrics on Hartogs domains, arXiv:0705.2124.

[12] G. Roos, A. Wang, W. Yin and L. Zhang, The Kähler-Einstein metric for some Hartogs domains over symmetric domains, Sci. China Ser. A 49 no. 9 (2006), 1175-1210.

Dipartimento di Matematica, Politecnico di Torino,

Corso Duca degli Abruzzi 24, 10129 Torino, Milano, Italy,

E-mail: antonio.discala@polito.it

Dipartimento di Matematica e Informatica Università di Cagliari, Via Ospedale 72, 09124 Cagliari, Italy,

E-mail: loi@unica.it

Dipartimento di Matematica e Informatica Università di Cagliari, Via Ospedale 72, 09124 Cagliari, Italy,

E-mail: fzuddas@unica.it 